Y. C. Minh and E. F. van Dishoeck, eds.

\title{
Excitation of $\mathrm{H}_{2}$ and HD in Shocks and PDRs
}

\author{
F. Bertoldi ${ }^{1}$, B. T. Draine ${ }^{2}$, D. Rosenthal ${ }^{3}$, R. Timmermann ${ }^{3}$, S. K. \\ Ramsay Howat ${ }^{4}$, T. Geballe ${ }^{5}$, H. Feuchtgruber ${ }^{3}$, and S. Drapatz ${ }^{3}$ \\ 1 Max-Planck-Institut für Radioastronomie, D-53121 Bonn, Germany \\ 2 Princeton University Observatory, Princeton, NJ 08540, USA \\ 3 Max-Planck-Institut für extraterrestrische Physik, D-85740 Garching, \\ Germany \\ 4 Royal Observatory, Blackford Hill, Edinburgh, EH9 $3 H J$, UK \\ 5 Gemini Observatory, Hilo, HI 96720, USA
}

\begin{abstract}
Photodissociation regions (PDRs) and shocks give rise to conspicuous emission from rotationally and vibrationally excited molecular hydrogen. This line emission has now been studied with ISO and from the ground in great detail. A remarkable discovery has been that toward the Orion outflow and other shock-excited regions, the $\mathrm{H}_{2}$ level populations show a very high excitation component. We suggest that these high-excitation populations may arise from non-thermal pumping processes, such as $\mathrm{H}_{2}$ formation and high-velocity ion-molecule collision in partially dissociative shocks. In PDRs such as NGC 7023 however, formation pumping is always less important than fluorescent pumping.

We furthermore present two HD emission line detections toward Orion Peak 1. This enables the first comparison of the $\mathrm{H}_{2}$ and the HD excitation, which surprisingly turn out to be identical.
\end{abstract}

\section{Introduction}

Shocks and photodissociation regions show strong $\mathrm{H}_{2}$ emission which is detectable in the visible, near- and mid-infrared. Among the important $\mathrm{H}_{2}$ lines observed are those of pure rotational transitions in the vibrational ground state, at wavelengths between about 2.4 and $28 \mu \mathrm{m}$. This spectral range has been difficult to access from the ground, and only recently has the Infrared Space Observatory (ISO) opened up this window to sensitive spectroscopy. Much attention has focused on the pure rotational emission, because it provides important clues about the physical state of the emitting gas. ISO and ground-based observations were able to trace the populations of levels at high energy, up to near the dissociation limit at $4.5 \mathrm{eV}$ above the ground state. Excitation diagrams such as those shown in Figs. 1 or 4 indicate that in shocks and PDRs (see Wright, this volume), the higher energy levels typically show remarkably high excitation temperatures, of order 2000 to $3000 \mathrm{~K}$. Could this emission from high levels actually be thermally excited? Although in principle possible, the kinetic temperatures and gas densities required for their thermalization seem 

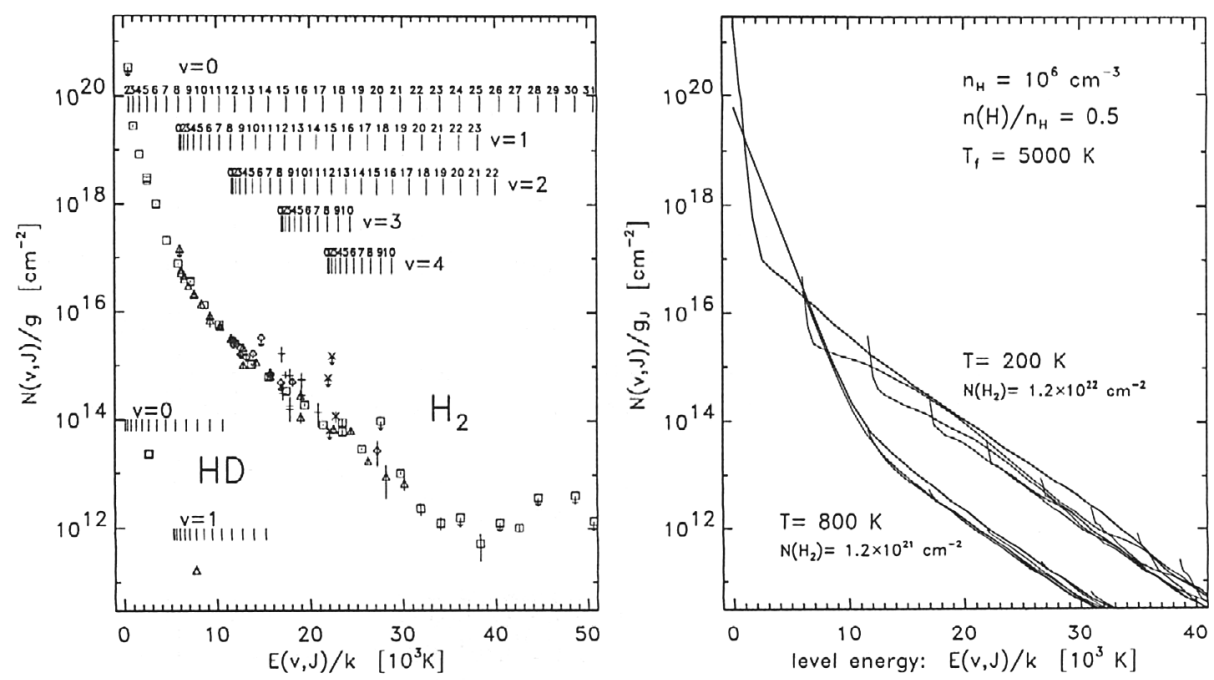

Figure 1. Left: Extinction-corrected, observed $\mathrm{H}_{2}$ and HD level column densities toward Orion Peak 1, divided by their degeneracy, plotted against the level energy $E(v, J)$. Right: Level populations predicted by non-LTE models of two gas layers with the labeled temperatures and column densities. Individual vibrational level distributions are shown as separate lines. The sum of the two distributions well matches the observed level distribution toward Peak 1 shown in the left panel.

uncomfortably high. What then could be the cause of this high excitation? In the following, we will briefly discuss several possible mechanisms.

\section{2. $\mathrm{H}_{2}$ in Shocks: the Orion Outflow}

OMC-1, the molecular cloud behind the Orion M42 Nebula, is the best-studied massive star-forming region. It embeds a young stellar object which produces a spectacular outflow that shocks the surrounding molecular gas, producing strong $\mathrm{H}_{2}$ infrared line emission fragmented in a multitude of clumps and arcs (Schultz et al. 1998). Peak 1 (Beckwith et al. 1978) is the brighter of the two $\mathrm{H}_{2}$ emission concentrations of the outflow. Toward Peak 1 we obtained 2.4-45 $\mu \mathrm{m}$ spectra with ISO, which show over $60 \mathrm{H}_{2}$ ro-vibrational and pure rotational lines, the latter ranging from 0-0 S(1) to 0-0 S(25) (Rosenthal et al. 1999, 2000). The extinction-corrected level column density distribution derived from the observed line intensities is shown in Fig. 1 (left).

\subsection{Excitation of high-energy $\mathrm{H}_{2}$ levels toward Peak 1}

Only a fraction $\sim 10^{-3}$ of the total column density $1.9 \times 10^{21} \mathrm{~cm}^{-2}$ of warm $\mathrm{H}_{2}$ toward Peak 1 resides in high energy levels, $E(v, J) / k>10,000 \mathrm{~K}$. For these levels the distribution of column densities implies an excitation temperature, $T_{e x}$, of about $3000 \mathrm{~K} ; T_{e x}$ is defined by a fit to the column densities of the 
form $N(v, J) / g \propto \exp \left(-E(v, J) / k T_{e x}\right)$. If the observed $N(v, J)$ is decomposed as a sum of LTE distributions with a range of excitation temperatures, then we find that about $99 \%$ of all warm $\mathrm{H}_{2}$ has $T_{e x}<2000 \mathrm{~K}$, and $90 \%$ has $T_{e x}<1000$ $\mathrm{K}$ (see Rosenthal et al. 2000). If the distribution of excitation temperatures were to reflect the actual distribution of kinetic temperatures, such a steep distribution of temperatures would be difficult to reconcile with the expected smooth temperature profile of planar C-type shocks. In C-type shocks the gas temperature changes smoothly, and a large fraction of the warm gas is near the maximum temperature reached in the shock (Timmermann 1996). Even with a distribution of shocks of different velocities and a corresponding range of peak temperatures, an excitation temperature distribution like that found toward Peak 1 is difficult to construct, unless one were to combine shocks with very disparate filling factors, e.g., several percent at $40 \mathrm{~km} \mathrm{~s}^{-1}$, the rest at $20 \mathrm{~km}$ $\mathrm{s}^{-1}$. Even in bow shocks, which are often cited to match the observed excitation (Smith 1991), the velocity changes slowly with distance from the apex, and the observed distribution of temperatures would hardly be expected.

In dissociative J-type shocks (e.g. Hollenbach \& McKee 1989), the molecules are destroyed in the shock, and they reform in a postshock layer where the temperature has dropped much below $3000 \mathrm{~K}$, somewhat dependent on the $\mathrm{H}_{2}$ formation rate at higher temperatures, which is very uncertain (e.g. Bertoldi 1997). Dissociative J-shocks can therefore also not account for the high excitation $\mathrm{H}_{2}$ we observe.

Even if temperatures of $3000 \mathrm{~K}$ or more can be reached in nondissociative shocks, the higher $\mathrm{H}_{2}$ levels would remain sub-thermally excited unless the gas density is high enough that the collisional excitation and deexcitation rates are comparable to those for radiative decay. A "critical" gas density can be estimated for a given level as that density for which the total collisional deexcitation rate of the level equals its total radiative decay rate. We find that even at kinetic temperatures of $3000 \mathrm{~K}$, gas densities well above $10^{6} \mathrm{~cm}^{-3}$ would be needed to maintain the higher $v=0$ levels at populations resembling LTE. Since such high densities may not prevail in the shocked gas of the Orion outflow, we explore mechanisms other than thermal excitation that might account for high populations in the upper energy states.

\subsection{Time-dependent C-shocks}

When a high velocity outflow strikes dense molecular gas, and a C-type shock is first established, J-type shocks can temporarily form within the C-shock (Chièze et al. 1998; Flower \& Pineau des Forêts 1999). In such an embedded J-shock, a small column of molecular gas is briefly heated to high temperatures without being dissociated, and if the gas density is sufficiently high, this would produce a high-excitation tail in the column density distribution. The lifetime of the embedded J-shock is small, and the high-excitation populations are transient, unless shocks were continuously reforming. Embedded J-shocks may also form when a C-shock encounters a dense clump. Since the time scale for the fading of the high-excitation tail in newly forming C-shocks is somewhat smaller than the age of the Orion outflow, we should expect spatial variations in the upper level excitation. Whether such gradients exist is unknown, but it would provide an interesting test for the transient J-shock model. 


\section{3. $\mathrm{H}_{2}$ formation pumping}

$\mathrm{H}_{2}$ formation is one other mechanism to populate the high-energy $\mathrm{H}_{2}$ levels. Molecular hydrogen is believed to form on the surfaces of dust grains. Some fraction of the $4.5 \mathrm{eV}$ released during the formation of an $\mathrm{H}_{2}$ molecule is required to leave the grain, and the remainder is split between translation, rotation, and vibration of the new molecule.

Toward Peak 1 we found that a fraction $6.8 \times 10^{-4}$ of the total warm $\mathrm{H}_{2}$ column is in states with energy $E(v, J) / k \geq 10,000 \mathrm{~K}$. Could $\mathrm{H}_{2}$ formation in a steady state account for such a fraction of molecules in highly excited states? We can try a very simple estimate. The pumping rate due to formation pumping is equal to the $\mathrm{H}_{2}$ formation rate, $n(\mathrm{H}) n_{\mathrm{H}} R_{g r}$, where we adopt $R_{g r} \approx 5 \times 10^{-17}$ $\mathrm{cm}^{3} \mathrm{~s}^{-1}$ as the $\mathrm{H}_{2}$ formation rate coefficient per hydrogen nucleus. The radiative decay rate we estimate from a characteristic radiative lifetime of a level at $\sim 2 \mathrm{eV}$, which is $\sim 10^{6} \mathrm{sec}$, and we prolong this by an average number of $\sim 5$ transitions for the molecule to reach its ground state. Thereby defining an effective $A$ coefficient for the decay rate of highly excited states, $A_{x} \approx 2 \times 10^{-7} \mathrm{~s}^{-1}$, the population balance for the excited states writes

$$
R_{g r} n_{\mathrm{H}} n(\mathrm{H})=n_{x}\left(\mathrm{H}_{2}\right) A_{x},
$$

which yields an excited $\mathrm{H}_{2}$ fraction

$$
\frac{n_{x}\left(\mathrm{H}_{2}\right)}{n\left(\mathrm{H}_{2}\right)}=\frac{n(\mathrm{H})}{n\left(\mathrm{H}_{2}\right)} \frac{n_{\mathrm{H}} R_{g r}}{A_{x}}=5 \times 10^{-4}\left(\frac{n_{\mathrm{H}}}{10^{6} \mathrm{~cm}^{-3}}\right)\left(\frac{n(\mathrm{H})}{2 n\left(\mathrm{H}_{2}\right)}\right),
$$

that agrees well with the observed value. This simple estimate shows that $\mathrm{H}_{2}$ formation could in principle account for some of the high-excitation level populations, provided the density is high enough, the atomic fraction is not small, and the formation rate coefficient in the warm shocked gas is comparable to the value implied at $\approx 100 \mathrm{~K}$ from Copernicus observations, $R_{g r} \approx 3 \times 10^{-17} \mathrm{~cm}^{3} \mathrm{~s}^{-1}$ (Jura 1975).

Can $\mathrm{H}_{2}$ formation also produce the observed shape of the high energy level populations?

The energy required to desorb from the dust grain depends on whether the atoms are bound by van der Waals forces (physisorbed, $\sim 0.03 \mathrm{eV}$ ), such as on water ice, or by chemical bonds (chemisorbed, $\sim 2 \mathrm{eV}$ ), like on a silicate surface. The exact level distribution of newly formed $\mathrm{H}_{2}$ is unknown, but it could very well contribute to the observed excitation at intermediate energies, $E(v, J) \approx 1-3 \mathrm{eV}$. Experiments and theoretical calculations are yet inconclusive regarding the excitation of a newly formed, desorbed hydrogen molecule. Hunter \& Watson (1978) consider physisorption and find a partition between rotational, vibrational, and translational energy, $E_{r} \approx 0.8 \mathrm{eV}, E_{v} \approx 3.3 \mathrm{eV}, E_{t} \approx 0.8 \mathrm{eV}$. Duley \& Williams (1986) assume the hydrogen to be physisorbed on silicate surfaces and find $E_{r}<0.01 \mathrm{eV}, E_{v} \approx 3.3 \mathrm{eV}, E_{t} \approx 0.2 \mathrm{eV}$. Duley \& Williams (1993) find that on PAH and graphite surfaces, $E_{r}<0.01 \mathrm{eV}, E_{v}<10^{-3} \mathrm{eV}$, whereas Duley \& Williams (1993) and Leonas \& Pjarnpuu (1981) find that on $\mathrm{H}_{2} \mathrm{O}$ ice and polymeric hydrocarbon surfaces, $E_{v} \approx 4 \mathrm{eV}$. Evidently, there is no conclusive agreement yet, except for a trend to expect that newly formed molecules must be vibrationally hot and rotationally cold. 
Using the PDR codes of Draine \& Bertoldi (1996), we computed the nonLTE level populations of partially dissociated gas of fixed density and temperature, and assuming that the $\mathrm{H}_{2}$ formation distribution follows

$$
\phi(v, J)=\phi(0,0) g_{n}(J) g_{f} e^{-E(v, J) / k T_{f}}
$$

where the nuclear degeneracy factor, $g_{n}(J)=1$ for even $J, 3$ for odd $J$. The formation temperature, $T_{f}$, is a free parameter, and so is the formation degeneracy, $g_{f}$. The exponential form of the level distribution (Black \& van Dishoeck 1987) is somewhat arbitrary, but in lack of observational constraints, seems reasonable. Fig. 2 shows the computed level populations. The high excitation tail in the population of levels with energy $>5000 \mathrm{~K}$ is due to formation pumping, whereas the lower levels are populated through collisional excitation. The difference in the level population when adopting $g_{f}=2 J+1$ or $g_{f}=1+v$ is illustrated in Fig. 2 (left). When emphasizing vibrational excitation, the individual vibrational level populations show a splitting similar to that seen for fluorescent excitation. When the weight lies on rotational excitation, the level populations remain tighter, more like what we observe toward Peak 1. Fig. 2 (right) shows how the level populations depend on the value of the formation temperature, $T_{f}$, where we adopt $g_{f}=2 J+1$.

No single temperature gas slab is able to reproduce the level distributions observed toward Peak 1. However, the sum of two components with hydrogen nuclei density $n_{\mathrm{H}}=10^{6} \mathrm{~cm}^{-3}$, atomic fraction $n(\mathrm{H}) / n_{\mathrm{H}}=0.5$, and respective temperatures of $200 \mathrm{~K}$ and $800 \mathrm{~K}$, with column densities $N_{\mathrm{H}_{2}}=1.2 \times 10^{22} \mathrm{~cm}^{-2}$ and $1.2 \times 10^{21} \mathrm{~cm}^{-2}$, can reproduce the observed level column distribution very well (Fig. 1, right). In order to produce a narrow distribution of level columns in the Boltzmann plot, we would have to assume pronounced rotational excitation of the newly-formed $\mathrm{H}_{2}$. This is somewhat contrary to the expectation of vibrationally hot, but rotationally cold new molecules.

\subsection{Non-thermal collisions}

An even more important pumping mechanism for the high-excitation levels could be non-thermal collisions between molecules and ions in a magnetic shock. In magnetic C-type shocks, which are believed to be responsible for most of the emission in Peak 1, the gas is accelerated through fast inelastic collisions. In a magnetic precursor the ions, which are tied to the magnetic field, collide with the undisturbed preshock gas at relative velocities comparable to the shock speed. Such non-thermal ion-molecule collisions lead to the acceleration of the molecules and to their internal excitation. High-velocity molecules subsequently collide with other molecules, resulting in a cascade of collisions during which the relative kinetic energy is in part converted to internal excitation of the molecules (O'Brien \& Drury 1996). In sufficiently fast C-shocks, the ion- $\mathrm{H}_{2}$ and $\mathrm{H}_{2}-\mathrm{H}_{2}$ collisions can lead to a significant collisional dissociation rate. The molecules dissociated in a partially dissociative, steady-state shock reform further downstream, so that across such a shock the $\mathrm{H}_{2}$ dissociation rate equals the $\mathrm{H}_{2}$ reformation rate. For every collisionally dissociated molecule there is likely to be a much larger number of inelastic collisions which did not lead to dissociation, but to the excitation of the molecule into high rovibrational states. 

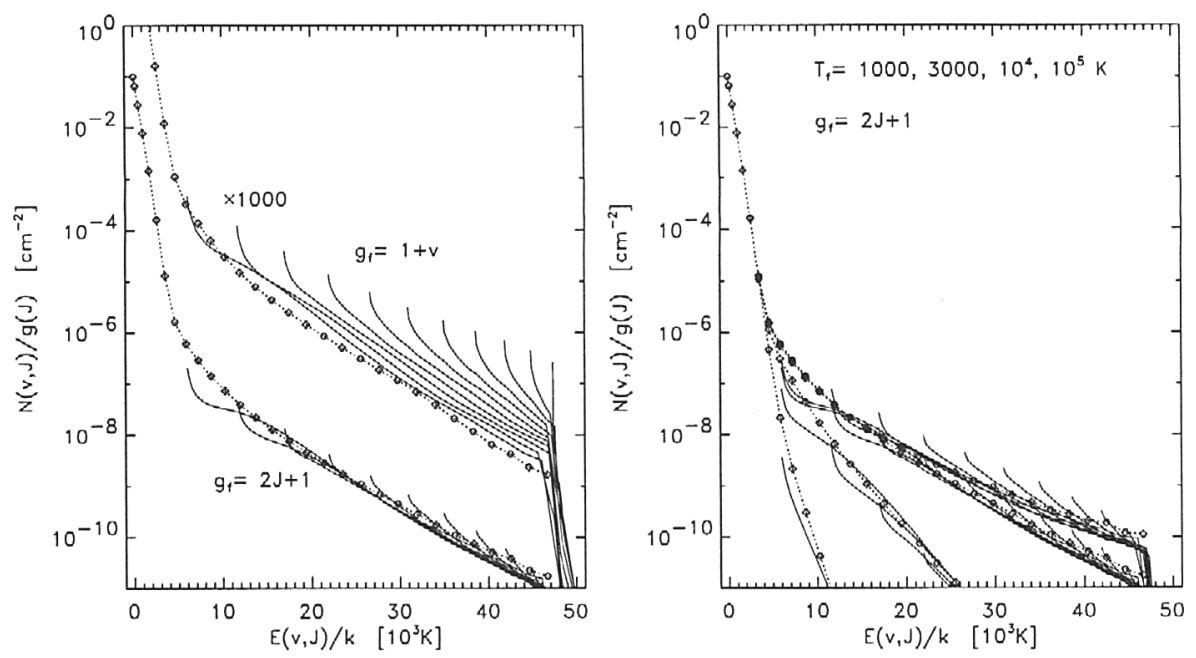

Figure 2. Level populations for non-LTE models including collisional processes and $\mathrm{H}_{2}$ formation, assuming a gas temperature of $400 \mathrm{~K}$, a number density of $\mathrm{H}$ nuclei of $10^{5} \mathrm{~cm}^{-3}$, and an atomic fraction of 0.5 , i.e., $n(\mathrm{H})=2 n\left(\mathrm{H}_{2}\right)$. Left: Two different distributions are assumed for newly-formed $\mathrm{H}_{2}$, one prefering vibrational, the other rotational excitation. Right: Comparison of level populations resulting from different $\mathrm{H}_{2}$ formation temperatures.

The high-excitation $\mathrm{H}_{2}$ level column densities thereby created should then be larger than those caused by $\mathrm{H}_{2}$ formation alone.

We conclude that non-thermal collisions in partially dissociative shocks could pump the high-excitation states in the $\mathrm{H}_{2}$ electronic ground state to the levels observed. No detailed shock models are available yet which account for this process.

\section{5. $0-0 \mathrm{~S}(25)$}

The $J=27$ level observed through the $0-0 \mathrm{~S}(25)$ line (Fig. 3) appears overpopulated by a factor of seven (see Fig. 1) over what would be expected from an extrapolation of the lower energy level populations. The $J=27$ level lies $3.6 \mathrm{eV}$ above ground, only $0.9 \mathrm{eV}$ from the dissociation limit. $\mathrm{H}_{2}$ that is newly formed on grains is less likely to populate rotational states so high, as some fraction of the formation energy is lost to overcome the grain surface potential, and some goes into vibrational excitation and translational kinetic energy.

Unless we misidentified the 0-0 S(25) line, it appears that a different mechanism may be populating this level, and possibly other high levels. The gas phase formation of $\mathrm{H}_{2}$ via $\mathrm{H}^{-}$, e.g., might be able to leave the new molecule in such a high rotational state. 


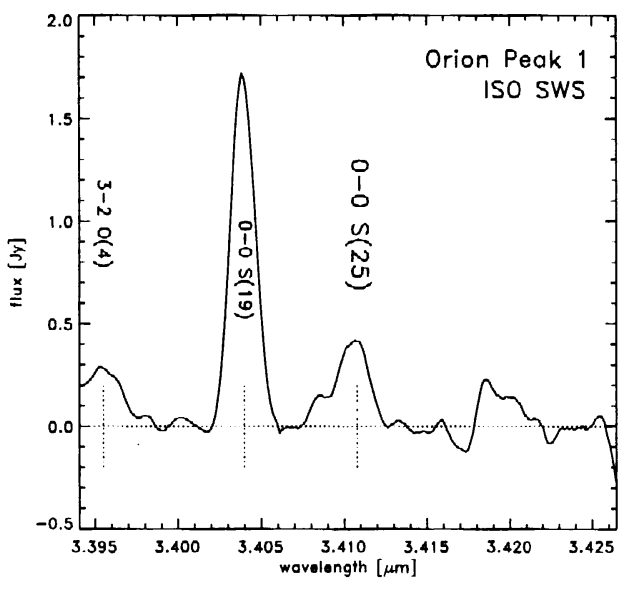

Figure 3. $\quad \mathrm{H}_{2} \quad v=0-0 \quad \mathrm{~S}(25)$ observed with the ISO SWS toward Orion Peak 1. The line appears clearly in this spectrum taken in the AOT $02, \sim 0.01 \lambda$ range grating scan mode, and also appears in the shallower AOT 01 full (2.4$45 \mu \mathrm{m})$ scan not shown here (see Rosenthal et al. 2000).

\section{Excitation of $\mathrm{H}_{2}$ in PDRs}

A "photodissociation region" is the interface separating a region which is predominantly molecular, and a region where the ultraviolet energy density is sufficiently high that hydrogen is mostly atomic. Young $\mathrm{O}$ and $\mathrm{B}$ stars located near molecular clouds produce conspicuous PDRs. A number of general reviews of PDRs have appeared recently (Hollenbach \& Tielens 1997, 1999; Sternberg, Yan, \& Dalgarno 1998; Walmsley 1998), and the current status of PDR modeling with regard to ISO observations was discussed by Draine \& Bertoldi (1999).

The structure of a PDR is determined primarily by the attenuation of the far-ultraviolet $(6-13.6 \mathrm{eV})$ radiation field, as one moves from the ionization front into the PDR. The dominant process is the photodissociation of $\mathrm{H}_{2}$, the rate for which is determined by both $\mathrm{H}_{2}$ self-shielding (Draine \& Bertoldi 1996) and attenuation by dust.

The $(v, J)$ ro-vibrational excited states of $\mathrm{H}_{2}$ are populated by inelastic collisions, by UV pumping, and by formation on grains. At the typical densities $n_{\mathrm{H}} \gtrsim 10^{4} \mathrm{~cm}^{-3}$ of bright PDRs, collisions maintain the $(v=0, J)$ levels of $\mathrm{H}_{2}$ in approximate thermal equilibrium for $J \lesssim 9$. Therefore measurements of the quadrupole emission line intensities with ISO provide a good indicator of the gas temperature. ISO observations toward the PDRs in, e.g., S140 (Timmermann et al. 1996), NGC 7023 (Fuente et al. 1999), or NGC 2023 (Draine \& Bertoldi 2000 ), provided unequivocal evidence for gas temperatures in the $500-1000 \mathrm{~K}$ range in a portion of the photodissociation region (PDR) where the $\mathrm{H}_{2}$ fraction is appreciable. These temperatures were higher than expected from current models of the heating and cooling processes in PDRs, and therefore require reconsideration of the physics of the gas and dust in PDRs.

The higher energy $\mathrm{H}_{2}$ levels are not populated by collisions, but by the decay of electronically excited states, which were pumped through the absorption of FUV photons. Because most molecules are excited from their ro-vibrational ground states, most subsequent transitions change the rotational quantum number, $J$, only by one or two, and the decay back into the electronic ground state populates states within a limited range of $J$. The cascade down the vibrational 


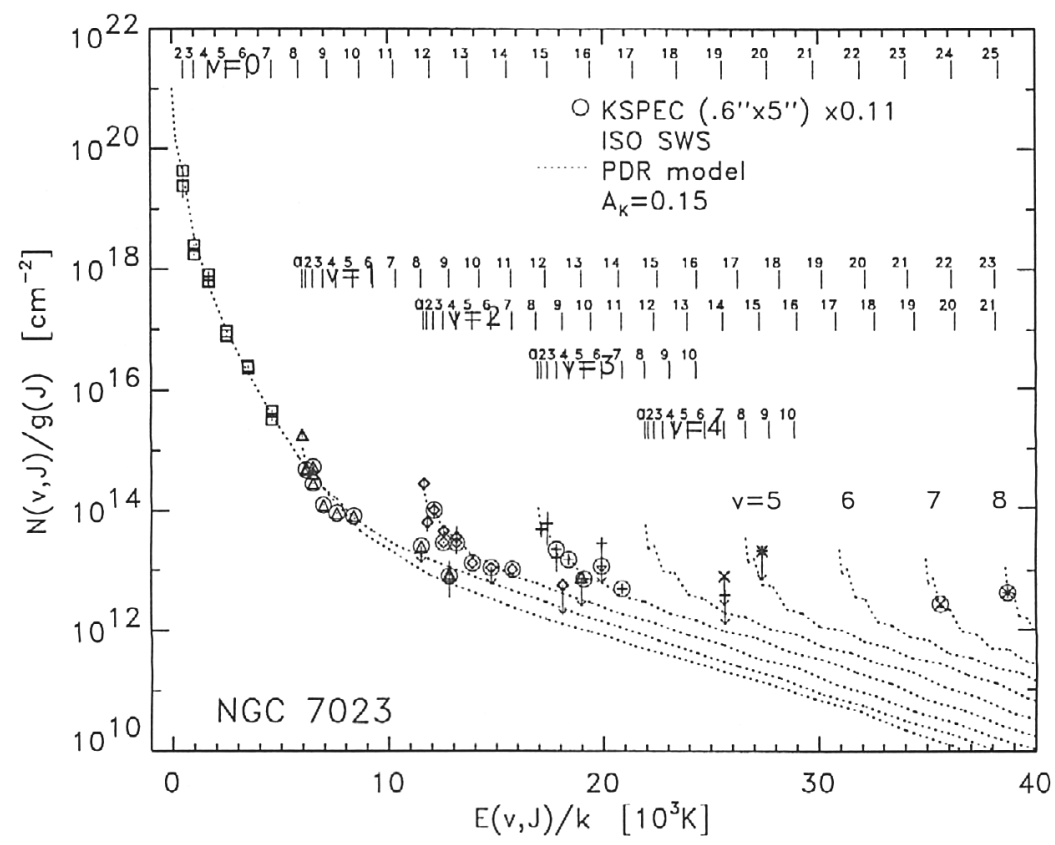

Figure 4. Extinction-corrected $\mathrm{H}_{2}$ level populations observed toward NGC 7023, plotted against the level energy. ISO and ground-based near-IR observations (Martini et al. 1997; circled symbols), scaled to match the ISO populations, are shown. For $v=0, J=2-7$ two ISO data points are shown: the respective upper ones are adopted from Fuente et al. (1999). Our PDR model for the level populations is traced by dotted lines.

states to the ground states can then significantly populate states up $J=10$ or beyond. Because $J=0,1$ pose a reflective boundary for the cascade down, the lowest $J$ levels within each vibrational state build up enhanced populations, which results in the characteristic population distribution of fluorescently excited $\mathrm{H}_{2}$ displayed in Fig. 4.

As an example we compare the level populations observed toward a bright filament in the NGC 7023 PDR with our best fit PDR model, which adopts $n_{\mathrm{H}}=10^{5} \mathrm{~cm}^{-3}$ and an incident FUV flux $\chi=5000$ times $1.2 \times 10^{7} \mathrm{~cm}^{-2} \mathrm{~s}^{-1}$. The observations shown include our ISO as well as ground-based spectroscopy. Our PDR model includes all basic collisional and radiative processes, including detailed thermal balance (Draine \& Bertoldi 1996,1999, 2000). The agreement between the model and the observations is remarkably good considering the simplicity of a constant density, planar slab model.

Black \& van Dishoeck (1987), Le Bourlot et al. (1995), and Bertoldi (1997) questioned whether processes other than collisions and fluorescence could affect the high energy or high rotational state populations in PDRs. Burton et al. (1992) suggested that $\mathrm{H}_{2}$ formation might be responsible for an apparent excess in the $v=4$ levels observed in the NGC 2023 PDR. 
Would we expect formation pumping to have a significant effect on the highexcitation level populations in PDRs? Of all photo-excitations of $\mathrm{H}_{2}$ by FUV photons, about 10 to $15 \%$ lead to dissociation, the remaining to pumping of high-excitation levels in the electronic ground state. In equilibrium, dissociation is balanced by reformation of $\mathrm{H}_{2}$, so that the ratio of the rates of formation pumping and fluorescent pumping of the high-excitation levels in the electronic ground state is $\sim 15 / 85$. Overall, fluorescent pumping should therefore dominate over formation pumping by a factor five. Unless the level distribution, $\phi(v, J)$, of newly formed $\mathrm{H}_{2}$ is strongly concentrated toward a small number of states (see Black \& van Dishoeck [1987] for models with pumping of specific levels), there will be little chance to detect signs of it in the level distributions of $\mathrm{H}_{2}$ in PDRs.

However, we should emphasize that the value of the $\mathrm{H}_{2}$ formation rate coefficient, $R_{g r}$, does strongly affect the total emission from a PDR, since it governs its molecular abundance structure, and thereby the fraction of the incident FUV energy that is reemitted by $\mathrm{H}_{2}$, as opposed to dust.
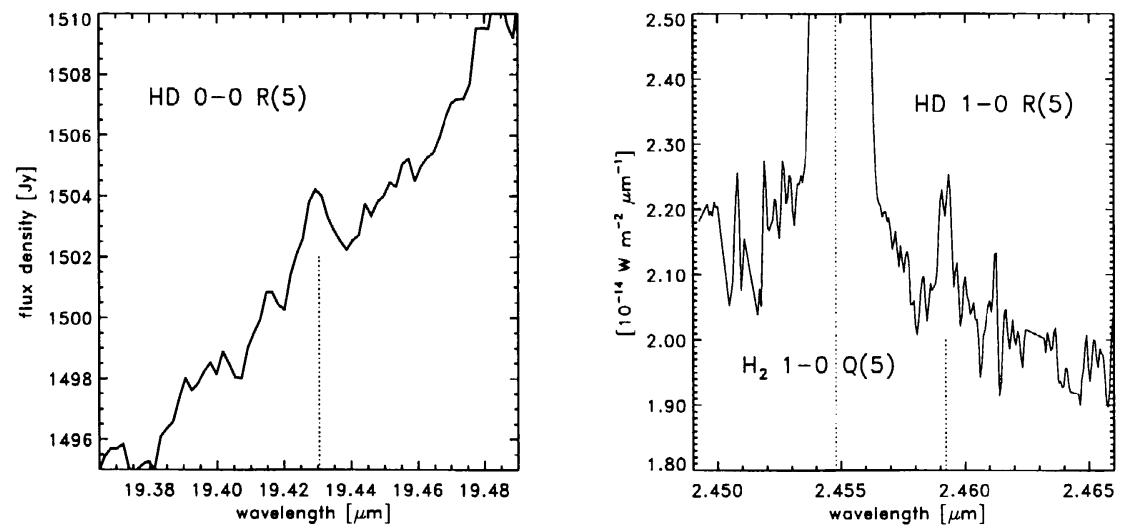

Figure 5. Detection of interstellar HD emission, obtained with the ISO SWS (left) and the UKIRT CGS4 (right) toward Orion Peak 1.

\section{HD Emission from the Orion Outflow}

The ideal molecule for measuring the interstellar deuterium abundance is HD, whose low rotational and ro-vibrational transition lines lie in the near- and midinfrared. We used ISO and the United Kingdom Infrared Telescope (UKIRT) to search for HD lines toward Peak 1. With ISO we there detected one faint HD line, 0-0 $\mathrm{R}(5)$ at $19.4 \mu \mathrm{m}$ (Fig. 5, Bertoldi et al. 1999), and with UKIRT we subsequently found the $1-0 \mathrm{R}(5)$ line at $2.46 \mu \mathrm{m}$, providing the first observation of $\mathrm{HD}$ emission with a ground-based telescope, and the first measurement of the rotation-vibrational excitation of HD in the ISM (Ramsay Howat et al., in prep.).

Surprisingly, we found that the HD excitation traced by the two observed transitions exactly mirrors the excitation of $\mathrm{H}_{2}$, despite the fact that the much 
faster radiative transitions of HD place the critical densities of its vibrationally excited states significantly above those of $\mathrm{H}_{2}$, and much higher than the estimated gas density in the outflow region. The cause for the similarity of the $\mathrm{H}_{2}$ and HD excitation is unresolved. It may imply a strong role of non-thermal excitation mechanisms, such as the direct coupling of the HD excitation to that of $\mathrm{H}_{2}$ through the exchange reaction $\mathrm{H}_{2}+\mathrm{D} \rightleftharpoons \mathrm{HD}+\mathrm{H}$. Current shock models are unable to explain the observed excitation of $\mathrm{HD}$.

The similarity of the $\mathrm{HD}$ and the well-traced $\mathrm{H}_{2}$ excitation permits the yet most accurate determination of the $[\mathrm{HD}] /\left[\mathrm{H}_{2}\right]$ abundance ratio in the warm, shocked gas, and with a $30 \%$ correction for chemical depletion of $\mathrm{HD}$ relative to $\mathrm{H}_{2}$ (due to an asymmetry in the above-mentioned exchange reaction - see Bertoldi et al. [1999], Ramsay Howat et al. in prep.) we are able to estimate a deuterium abundance $[\mathrm{D}] /[\mathrm{H}]=(5.1 \pm 1.5) \times 10^{-6}$.

This value is low compared with previous $\mathrm{D}$ and $\mathrm{H}$ absorption measurements towards nearby stars, which found values higher by a factor of two to three. But it is still consistent with two other measurement of the deuterium abundance towards Orion (Wright et al. 1999; Jenkins et al. 1999). This indicates that in the Orion region, either stars have burnt up a large fraction of the primordial deuterium, or more likely, that there is significant trapping of deuterium on dust grains. Future observations from space and from the SOFIA airborne observatory will eventually tell.

Acknowledgments. We are thankful to G. Pineau des Fôrets, J. Black, E. van Dishoeck, and C. Wright for valuable input, and to the SWS Data Center at MPE, especially to E. Wieprecht, for their support. BTD was supported in part by NSF grant AST-9619429.

\section{References}

Beckwith, S., Persson, S., Neugebauer, G., \& Becklin, E. 1978, ApJ, 223, 464

Bertoldi, F. 1997, in Proceedings of the first ISO workshop on Analytical Spectroscopy, eds. A.M. Heras et al. (Noordwijk: ESA Publications Division, ESA SP-419), 67

Bertoldi, F., Draine, B.T., Drapatz, S., Rosenthal, D., Timmermann, R., \& Wright, C.M. 1999, in The Physics and Chemistry of the Interstellar Medium, eds. V. Ossenkopf et al. (GCA-Verlag Herdecke)

Bertoldi, F., Timmermann, R., Rosenthal, D., Drapatz, S., \& Wright, C.M. 1999, A\&A, 346, 267

Black, J. \& van Dishoeck, E.F. 1987, ApJ, 322, 412

Burton, M., Bulmer, M., Moorhouse, A., Geballe, T.R., \& Brand, P.W.J.L. 1992, MNRAS, 257, 1

Chièze, J.-P., Pineau des Forêts, G., \& Flower, D. 1998, MNRAS, 295, 672

Draine, B.T. \& Bertoldi, F. 1996, ApJ, 468, 269

1999 , in The Universe as seen by ISO, eds. P. Cox \& M.F. Kessler (ESA SP-427)

2000, in $\mathrm{H}_{2}$ in Space, eds. F. Combes \& G. Pineau des Forêts, Cambridge University Press

Duley, W.W. \& Williams, D.A. 1986, MNRAS, 223, 177

1993, MNRAS, 260, 37 
Flower, D. \& Pineau des Forêts, G. 1999, MNRAS, 308, 271

Fuente, A., Martín-Pintado, J., Rodriguez-Fernandez, N.J., de Vicente, P., \& Kunze, D. 1999, ApJ, 518, L45

Hollenbach, D.J. \& McKee, C.F. 1989, ApJ, 342, 306

Hollenbach, D.J. \& Tielens, A.G.G.M. 1997, ARA\&A, 35, 179 1999, Rev. Modern Phys., 71, 173

Hunter, D.A. \& Watson, W.D. 1978, ApJ, 226, 477

Jenkins, E., Tripp, T., Wozniack, P., et al. 1999, ApJ, 520, 182

Jura, M. 1975, ApJ, 197, 575

Leonas, V.B. \& Pjarnpuu, A.A. 1981, Sov. Ast. Let., 7, 19

Le Bourlot, J., Pineau des Forêts, G., Roueff, E., Dalgarno, A., \& Gredel, R. 1995, ApJ, 449, 178

Martini, P., Sellgren, K., \& Hora, J.L. 1997, ApJ, 484, 296

O'Brien, I. \& Drury, L. 1996, MNRAS, 280, 550

Rosenthal, D., Bertoldi, F., Drapatz, S., \& Timmermann, R. 1999, in The Universe as seen by ISO, eds. P. Cox \& M.F. Kessler (ESA SP-427), 561

Rosenthal, D., Bertoldi, F., \& Drapatz, S. 2000, A\&A, submitted

Smith, M. 1991, MNRAS, 253, 175

Schultz, A.S.B, Colgan, S.W.J., Erickson, E.F., et al. 1999, ApJ, 511, 282

Sternberg, A., Yan, M., \& Dalgarno, A. 1998, in Molecules in Astrophysics: Probes and Processes, ed. E.F. van Dishoeck (Kluwer: Dordrecht), 141

Timmermann, R., Bertoldi, F., Wright, C.M., Drapatz, S., Draine, B.T., Haser, L., \& Sternberg, A. 1996, A\&A, 315, L281

Timmermann, R. 1996, ApJ, 456, 631

Walmsley, C.M. 1998, Astr. Lett. \& Comm., 37, 1

Wright, C.M., van Dishoeck, E.F., Cox, P., Sidher, S., \& Kessler, M.F. 1999, ApJ, 515, L29 


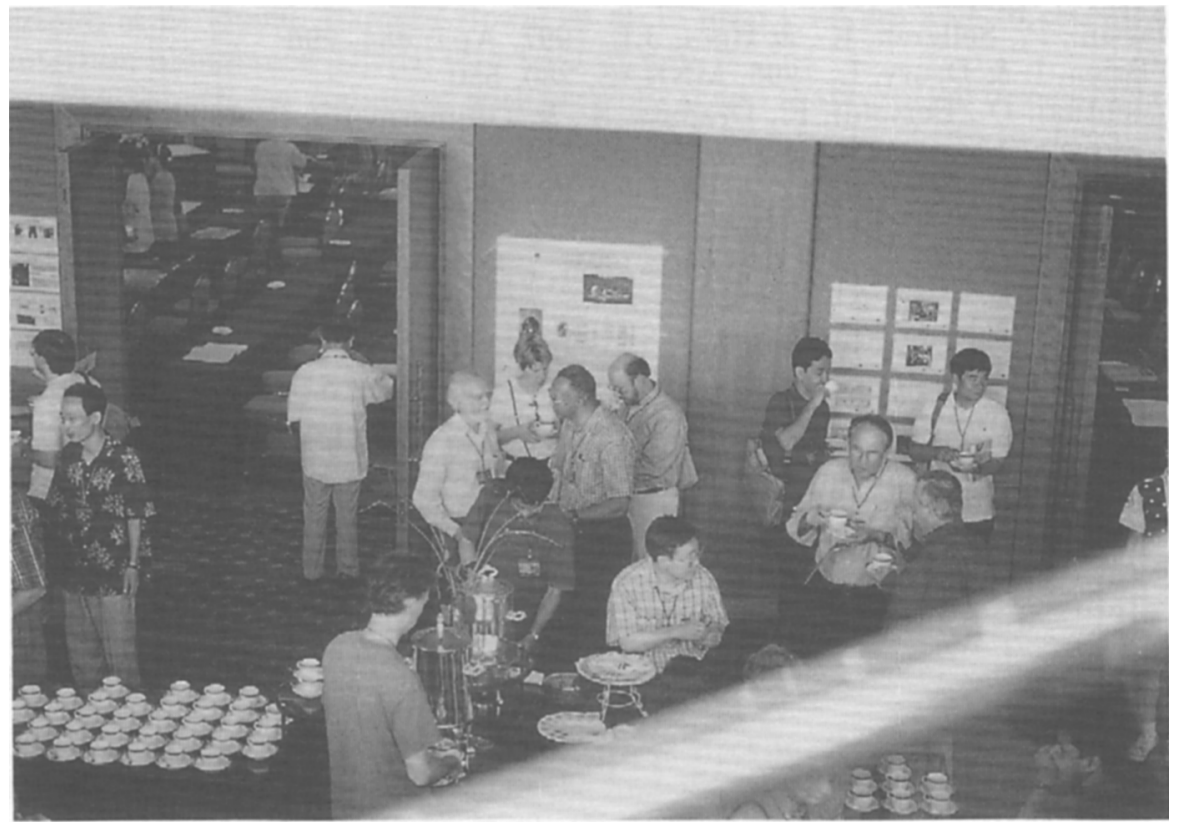

